\title{
Lateral medullary infarction extending to the lower pons
}

\author{
C M FISHER, J TAPIA \\ From the Neurology and Neuropathology Services of the Massachusetts General Hospital, Boston, USA
}

SUMMARY A case of lateral ponto-medullary infarction was studied pathologically using serial sections of the involved region. The infarct involved $4 \mathrm{~mm}$ of the lower pons and $10 \mathrm{~mm}$ of the upper medulla. The feeding arteries of most of the infarct were traced back to form one small artery $350 \mu \mathrm{m}$ in diameter which arose from an artery $2 \mathrm{~mm}$ in diameter identified as the posterior inferior cerebellar artery. The latter was occluded by a recent thrombus or embolus which blocked the mouth of the branch to ponto-medullary region.

Classical lateral medullary infarcts most commonly occupy the middle one third of the medulla with extension superiorly or inferiorly. ${ }^{1}$ Occasionally, only the upper one third of the medulla is involved in which case the infarct may extend upwards into the lateral tegmentum of the pons producing signs of 6 th, 7th, or 8th cranial nerve deficits. Mild facial weakness is often remarked on in cases of lateral medullary infarction; Isch ${ }^{2}$ for example observed a partial nuclear palsy in five cases and a simple hypotonia in 14 , in a series of 39 cases. In another series ${ }^{1}$ an ipsilateral weakness was noted in 12 of 18 cases. Severe facial palsy of a peripheral type, which would clearly indicate pontine involvement is infrequent. The present paper is the report of a clinico-pathologic study in which not only was the location of the infarct determined by serial sections, but the underlying vascular mechanism was demonstrated to be obstruction of the branch artery-of-supply by a thrombus or embolus lying in the parent posterior inferior cerebellar artery (PICA).

\section{Case report}

A 71-year-old hypertensive woman with chronic pulmonary emphysema suddenly without prodromal symptoms developed vertigo, nausea, repeated vomiting, dysphagia, and inability to walk. She also described diplopia with images diagonally separated, numbness of the lips, left side of face and right arm, and at the onset of the stroke an

Address for reprint requests: Dr C Miller Fisher MD, Neurology Service, Massachusetts General Hospital, Fruit St, Boston, Mass. 02114, USA.

Received 15 April 1986. Accepted 5 June 1986 intermittent roaring in the left ear. Her sister immediately noted drooping of the left side of the face and dysarthria.

On examination in the emergency department during the next several hours, she was alert, animated, and correctly oriented. There was an obvious severe left facial paralysis of a peripheral type. Dysarthria was so severe she could hardly be understood. The right pupil measured $3 \mathrm{~mm}$ in diameter. the left pupil $1.5 \mathrm{~mm}$ and they reacted to light. Both sides or the forehead were dry. The ocular movements were full with a slow coarse horizontal nystagmus to the right (con-? tralateral) on right lateral gaze and a fine shimmering nystagmus to the left (ipsilateral) on left lateral gaze. On gaze upwards and downwards there was a rotatory component. There was vertical and horizontal diplopia with the right eye, hyperopic. Hearing was decreased in the left ear compared with the right. The corneal reflex was diminished bilaterally, more on the right side. The gag reflex was decreased on the left side. The palate elevated bilaterally. The patient complained of difficulty in swallowing her saliva. The tongue deviated to the right on protrusion but was placed more strongly in the right cheek than the left.

Motor power in the limbs was intact. The tendon reflexes were $2+$ and equal on the two sides and the plantar responses were flexor. Sensation to pinprick and cold was diminished or absent on the entire right side (face, neck, $\mathrm{arm}$, and leg) whereas appreciation of vibration, touch, and joint position was correct. There was a slight instability on the finger-nose, and heel-knee-tibia test on the left side. Stance and gait were not tested.

The blood pressure was $210 / 100 \mathrm{~mm} \mathrm{Hg}$. An electrocardiogram showed normal sinus rhythm and borderline left axis deviation. Computed tomography (CT) of the head was essentially normal. With a tentative diagnosis of basilar artery stenosis, intravenous heparin therapy was begun.

Twenty four hours after admission, the patient's condition having remained stable otherwise, she complained of having difficulty getting her air. She was sufficiently alert to use a catheter herself to suction her throat after coughing. 
Six hours later she was cyanotic and complained of not getting enough air. Oxygen was administered by mask, but one hour later she was found pulseless. Resuscitation was complicated by difficulty in accomplishing intubation and the patient remained comatose. Immediately after resuscitation the pupils reacted to light, the eyeballs did not move on head rotation or ice-water irrigation of the ears, and stimulation evoked bilateral extensor posturing of the limbs. After 12 hours, reflex horizontal movements of the right eyeball were full while the left eye moved well medially but not laterally. There was widespread myoclonic jerking. Pinprick to the nostrils elicited no response. The patient died 10 days after cardiorespiratory arrest, having shown no response to verbal stimuli and only extensor posturing to painful stimuli. Myoclonic jerking had continued.

\section{Pathological findings}

a. Gross. There was only slight pulmonary congestion. The left ventricular wall of the heart was thickened and the atria were dilated. The brain superficially showed no lesions. The arteries at the base were severely atherosclerotic and the lumen of the basilar artery was narrowed to less than $0.5 \mathrm{~mm}$ at a point $15 \mathrm{~mm}$ above the junction of the vertebral arteries. The vertebral arteries were of good size and patent. The left PICA arose from the left VA $11 \mathrm{~mm}$ from the basilar artery and was patent in its proximal $8 \mathrm{~mm}$ at which point it disappeared from view. Both posterior communicating arteries were approximately $1 \mathrm{~mm}$ in diameter.

Upon sectioning, the hemispheres were intact except for two small recent infarcts approximately 9 by $4 \mathrm{~mm}$ symmetrically located one each side in the vicinity of the nucleus accumbens. The globus pallidus on each side and the cerebral cortex were intact. The watershed regions showed no gross damage. The midbrain and upper pons were normal.

For demonstration purposes, two knife-cuts were made in the lower brainstem starting posteriorly and reaching almost but not to the anterior surface, in this way avoiding disruption of the small arteries entering the brain stem from in front. One cut was at the level of the ponto-medullary junction and demonstrated a recent typical lateral medullary infarct on the left side; the second was made 3-4 mm above the first and demonstrated a similar softening in the comparable midportion of the pontine tegmentum on the left side. There was no evidence that the stenotic lesion of the basilar artery had caused ischaemic damage to the upper brainstem. The cerebellum was intact both deep and superficially. An attempt was made to identify grossly the small arteries running to the region of the infarction, but when this was not readily accomplished further dissection was abandoned lest small vessels be torn, interfering with the plan for serial sectioning. The anterior inferior cerebellar artery and its branches did not supply the region of the infarct. A block of tissue containing the ponto-medullary infarct and encompassing the lower $4 \mathrm{~mm}$ of the pons and the upper $1.0 \mathrm{~cm}$ of the medulla was prepared for serial sectioning. To simplify processing the right half of the pons and medulla and the left side of the pons lateral to the 8th nerve were cut away.

b. Microscopic. Sections stained with luxol fast blue and haematoxylin and eosin showed zones of recent laminar necrosis scattered throughout the cerebral cortex. The Purkinje cells of the cerebellar cortex were greatly reduced in number. The ischaemic-anoxic damage was surprisingly light considering the patient's greatly reduced clinical state in the days following cardiorespiratory arrest.

The ponto-medullary block of tissue was serial sectioned at $10 \mu \mathrm{m}$ and all 2000 sections were stained with the phosphotungstic acid haematoxylin stain. Although the preliminary gross knife-cuts marred the demonstration to some extent, the infarct could be satisfactorily delineated and the pattern of blood supply to its major portion traced back to a site of vascular occlusion.

The infarct consisted of a mass of fatty macrophages in keeping with an age of 10 days. Its vertical (rostral-caudad) extent was $14 \mathrm{~mm}$ of which the upper $4 \mathrm{~mm}$ lay in the pons and the lower $10 \mathrm{~mm}$ in the medulla. (The dividing line between the two was the inferior margin of exit of the facial nerve.) The medullary portion (fig 1 ) consisted of a $5 \times 5 \mathrm{~mm}$ column of softening in the classical location posterior to the inferior olivary nucleus and extending from the surface, medially and posteriorly to within $1.5 \mathrm{~mm}$ of the floor of the 4 th ventricle. The pontine portion (figs 2 and 3 ) was a direct upward or rostral continuation of the medullary infarction and occupied a comparable zone in the tegmentum. At the level of the 7th nerve exit, it measured $6 \mathrm{~mm}$ long and $3 \mathrm{~mm}$ wide and reached to within $1 \mathrm{~mm}$ of the floor of the 4th ventricle. Above this it gradually narrowed to $1.5 \mathrm{~mm}$ and abutted on the ventricular surface. The infarct lay just medial to the 7th nerve nucleus and lateral to the position of the 6th nerve nucleus which actually lay slightly rostral to the superior boundary of the infarct. The facial nerve was involved as it coursed medially and posteriorly to loop around the 6th nerve nucleus.

The Vascular supply Using the uninterrupted series of sections the small arteries supplying the medullary part of the infarct were traced retrogradely throughout the softening to their entrance into the subarachnoid space where they successively joined one another to finally form a single artery $350 \mu \mathrm{m}$ in diameter which was then followed back to its origin from an artery $2 \mathrm{~mm}$ in diameter probably the left PICA which itself was tightly occluded by a recent thrombus or embolus blocking the mouth-of-origin of the branch (fig 4). The smaller branches within the pontine portion of the infarct were traced back to a 


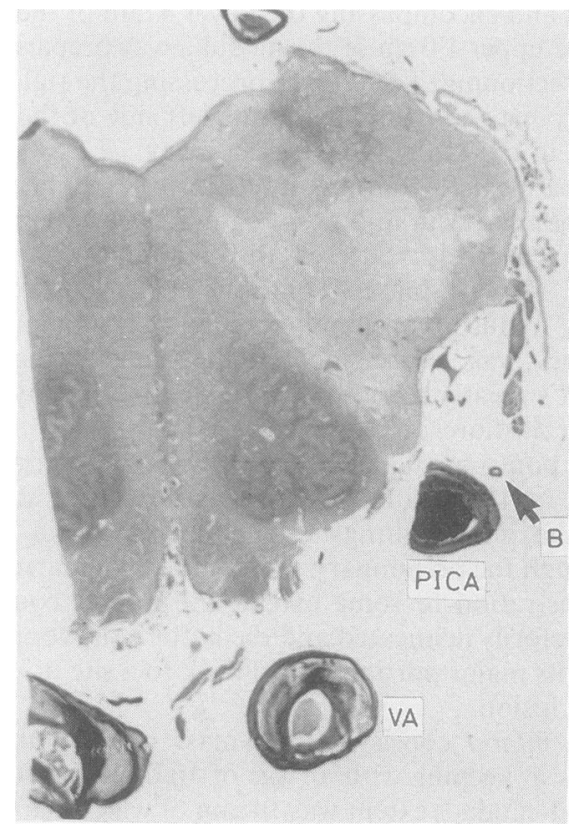

Fig 1 Infarct in medulla $(\times 2)$. VA, left vertebral artery; $P I C A$, left posterior inferior cerebellar artery containing thrombus; $B$, Branch to infarct.

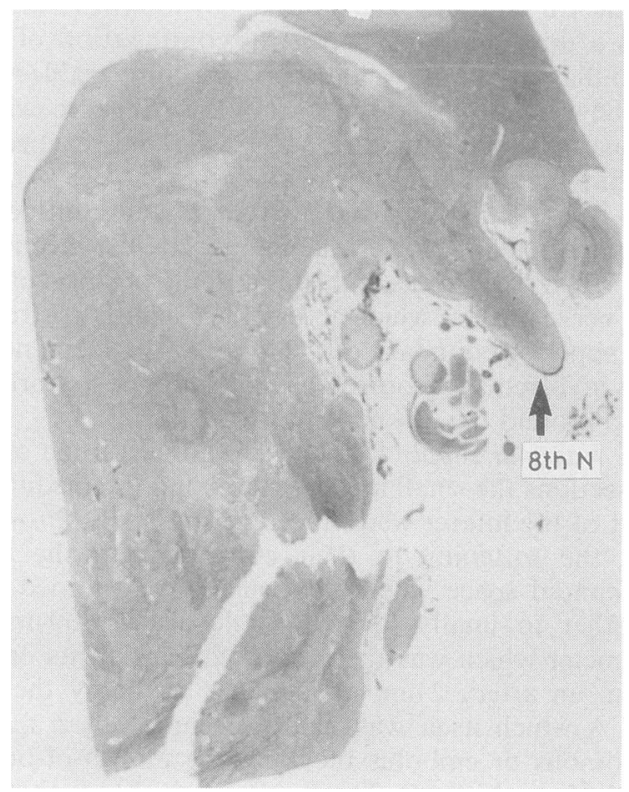

Fig 2 Infarct in lower pons at level of 8th nerve $(\times 2)$.

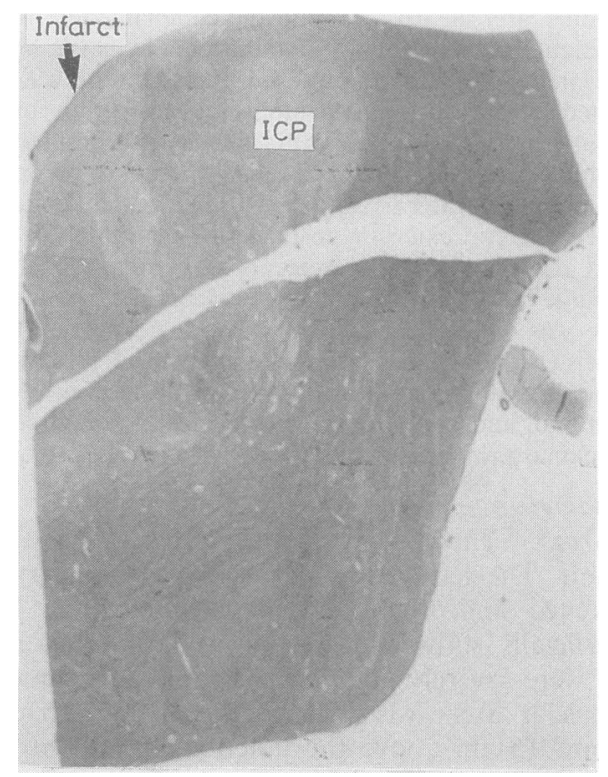

Fig 3 Uppermost part of infarct in lower pons $(\times 2)$. ICP, inferior cerebellar peduncle. Break in section is due to knife cut.

single small arterial branch which suddenly disap peared from the sections and whose more proximas end could not be identified. It lay at the upper end of? the line of small arteries to the medullary portion and it is reasonable to suggest it had the same origin. It probably had been severed inadvertently by the original preliminary knife-cut.

The main artery-of-supply of the infarcted territory was traced back unequivocally to its origin from the larger artery $2 \mathrm{~mm}$ in diameter. However, only a $4 \mathrm{~mm}$ segment of the latter vessel was included in the sections. It lay on the lateral aspect of the medulla in the usual position of the PICA and since both vertebral arteries were visible and accounted for in the sections, it was concluded that this vessel must have been the left PICA. It was tightly occluded by a thrombus which consisted of fibrin and platelets and extended into the mouth-of-origin of the artery of the infarct (fig 5). The wall of the occluded segment of artery showed only slight atherosclerosis suggesting that the obstructing clot was embolic rather than thrombusin-situ. This would be consistent with the sudden onset of the stroke without prodromal warning. The proximal $8 \mathrm{~mm}$ of the left PICA had been visible on the anterior medulla and was grossly free of clot. However, since the entire artery was not included in the examination the nature of the occlusion must remain uncertain. At the same time as the possibility 


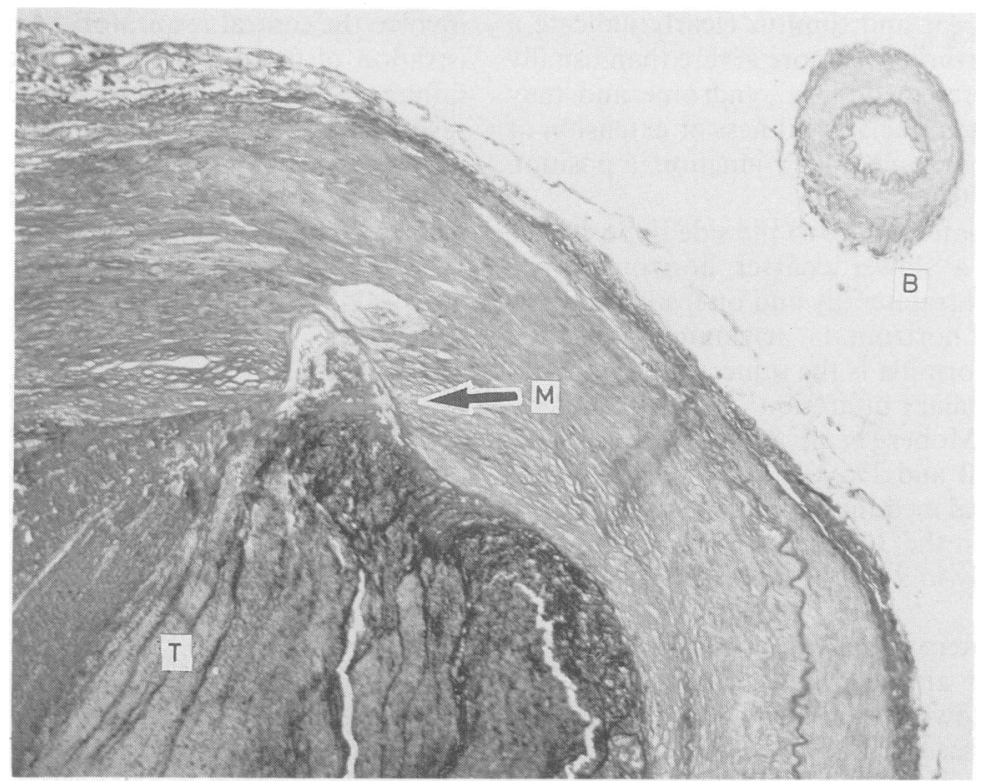

Fig 4 Recent thrombus (T) in PICA extending into mouth of branch (M) $(P T A H \times 64) . B$, branch to infarct.

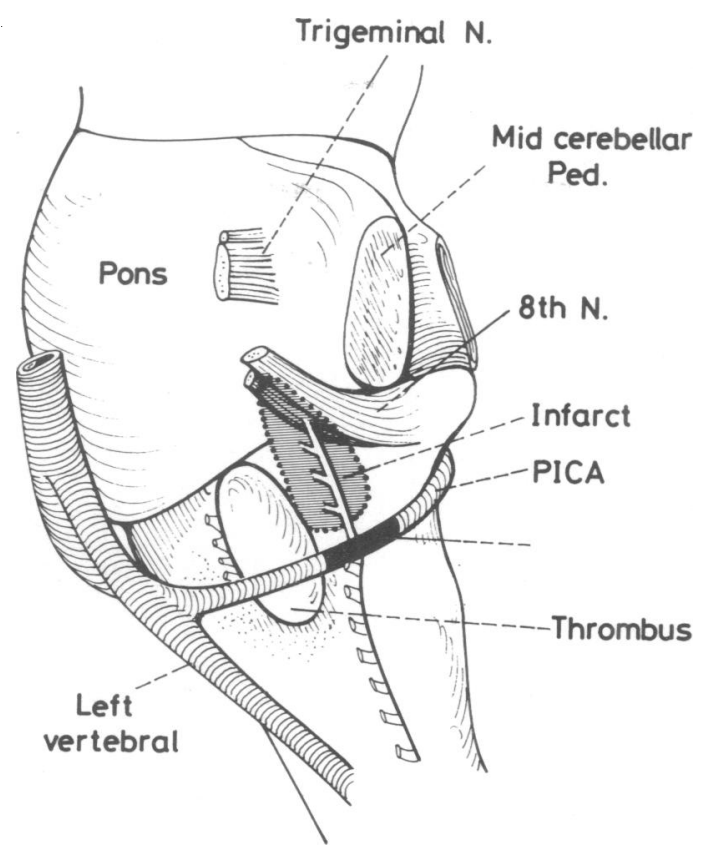

Fig 5 Diagram of lateral aspect of lower brainstem showing vascular arrangement. of embolism is mentioned, it should be added that no source of embolism was found post mortem, nor was there evidence of embolism in other organs. Although the PICA was tightly occluded the cerebellum in its territory of distribution was not infarcted.

\section{Discussion}

This case report is informative mainly in two ways: (1) It provides the anatomic basis for a lateral pontomedullary infarct, and (2) it illustrates one of the vascular mechanisms which may underlie such an infarct, namely acute occlusion of the parent PICA with blockage of the mouth of a branch which was accurately identified as the artery-of-supply to the territory of infarction. It is a good example of focal compromise of the circulation in a penetrating branch when its mouth is obstructed by thrombus in the parent vessel, and extends the observations on occlusion of penetrating branches of the basilar artery. ${ }^{34}$

A few points in the clinico-pathologic correlation warrant comment. Most of the clinical manifestations can be accounted for by the lateral medullary part of the infarct: vertigo, nausea, vomiting, diplopia, dysphagia, ipsilateral facial numbness, Horner's syndrome, nystagmus, skew deviation, contralateral loss of sensation to pin prick and ipsilateral cerebellar ataxia. The complete facial paralysis, diminished 
hearing in the left ear and tinnitus clearly indicate a pontine site. Dysarthria was more severe than usually occurs in the lateral medullary syndrome and may reflect the presence of facial weakness or extension of the infarct to the pontomedullary junction, a position more rostral than usual.

In this case, on lateral gaze to the side opposite the lesion there was a slower coarser horizontal nystagmus beating contralaterally and on ipsilateral gaze a faster fine horizontal nystagmus beating ipsilaterally. The formula is the same as in a previous case of pontomedullary infarction ${ }^{1}$ and in the lateral medullary case of Moberg et al, ${ }^{5}$ but the reverse of the case of Kemmerell and Hoyt. ${ }^{6}$ The pattern of nystagmus in the lateral medullary syndrome, however, is not uniform and in the 34 cases of Isch, ${ }^{2}$ nystagmus beat contralaterally in 25 cases and ispsilaterally in 9 cases.

The presence intermittently of a roaring sound in the ipsilateral ear at the onset of the stroke is a reminder that in lower brain stem ischaemia due to vertebro-basilar disease, and in other processes as well, assorted auditory hallucinations of a minor nature are not rare. In this case the lesion lay close to the cochlear nucleus and also was in a position to involve the trapezoid body.

The cause of respiratory failure remains uncertain. There was no evidence of extension of the infarct to involve the central respiratory apparatus and administration of $\mathrm{O}_{2}$ to a patient with chronic pulmonary failure and an elevated $\mathrm{pCO}_{2}$ was probably responsible.

This study was supported by a grant from the Freed Foundation, Washington, D.C.

\section{References}

1 Fisher CM, Karnes WE, Kubik CS. Lateral medullary infarction - the pattern of vascular occlusion. $J \mathrm{Neu}$ ropathol Exp Neurol 1961;20:323-78.

2 Isch F. Formes de début et séquelles du syndrome rétroolivaire de Dejerine. Rev Neurol (Paris) 1957;97: 350-65.

3 Fisher CM, Caplan LR. Basilar artery branch occlusion: A cause of pontine infarction. Neurology 1971;21: 900-5.

4 Fisher CM. Bilateral occlusion of basilar artery branches. Neurol Neurosurg Psychiatry 1977;40: $1182-9$.

5 Moberg A, Preber L, Silfverskiöld BP, Vallko S. Imbalance nystagmus and diplopia in Wallenberg's syndrome. Acta Laryngol (Stockh) 1962;55:269-82.

6 Kommerell G, Hoyt WF. Lateropulsion of saccadic eye movements. Electro-oculographic studies in a patient with Wallenberg's syndrome. Arch Neurol 1973;28. 313-8. 